\title{
Double Photoionization of excited Lithium and Beryllium
}

\author{
F. L. Yip, ${ }^{1}$ C. W. McCurdy, ${ }^{2,1}$ and T. N. Rescigno ${ }^{1}$ \\ ${ }^{1}$ Lawrence Berkeley National Laboratory, Chemical Sciences, \\ and Ultrafast X-ray Science Laboratory, Berkeley, CA 94720 USA \\ ${ }^{2}$ Departments of Applied Science and Chemistry, \\ University of California, Davis, CA 95616 USA
}

(Dated: May 20, 2010)

\begin{abstract}
We present total, energy-sharing and triple differential cross sections for one-photon, double ionization of lithium and beryllium starting from aligned, excited $\mathrm{P}$ states. We employ a recently developed hybrid atomic orbital/ numerical grid method based on the finite-element discrete-variable representation and exterior complex scaling. Comparisons with calculated results for the groundstate atoms, as well as analogous results for ground-state and excited helium, serve to highlight important selection rules and show some interesting effects that relate to differences between interand intra-shell electron correlation.
\end{abstract}

\section{INTRODUCTION}

Photo-double ionization (DPI) processes, in which two electrons are ejected into the continuum following absorption of a single photon, offer a powerful method for exploring the effects of electron correlation. The joint angular distribution provides the most sensitive probe of electron correlation, as well as the most significant challenges to $a b$ initio theory, since its accurate description cannot be obtained with independent particle models nor, at energies close to threshold, with perturbative methods. While DPI from simple closed-shell atoms has been relatively well studied, there has been far less theoretical work on DPI from excited states. To our knowledge, theoretical results for differential DPI cross sections from excited states have been calculated only for helium [1] and only for excited S states. Excited atomic targets with $L \neq 0$ can be aligned and then offer the possibility of studying the dependence of DPI cross sections on the direction of photon polarization, posing additional challenges to theory. Indeed, the first experimental measurements on the orientation dependence of DPI cross sections have recently been performed on laserexcited and aligned lithium [2]. These initial measurements only examined orientation dependence of the total cross sections, but given the continued rapid developments in trapping, laser-preparation and advanced detection techniques, coupled with the availability of intense VUV optical sources, it should not be long before results from kinematically complete experiments on DPI from excited atomic targets become available. With that anticipation in mind, we present the results of calculations on fully differential angular distributions for DPI from aligned lithium and beryllium atoms in excited P-states, along with comparisons to analogous processes in helium.

The results presented here were obtained with a a gridbased application of the exterior complex scaling (ECS) method [3], recently extended to handle many-electron targets [4]. This modification of the finite-element discrete variable representation (FEM-DVR) approach uses atomic orbitals, constructed from DVR functions in the first few elements, to describe the inner-shell electrons while the remainder of the DVR representation is used to represent the continuum portions of the wave function. This method, which was used in our recent study of DPI from ground-state beryllium [4], along with other aspects of the theoretical formulation, are briefly described in the following section. Computational details are then presented in Sec. III, followed by results for lithium and beryllium. We conclude with a brief summary.

\section{THEORETICAL METHOD}

The idea behind the hybrid orbital/FEM-DVR approach is conceptually simple. The goal is to derive an effective two-electron Hamiltonian that can be represented with an exterior-scaled FEM-DVR basis. To treat double continuum processes in an $\mathrm{N}$-electron atom with two "active" electrons, one expands the wave function in configurations in which two electrons are represented by a FEM-DVR product basis of radial functions $\chi$ and spherical harmonics $Y_{l m}$, and the rest are represented by atomic orbitals $\varphi$ with a fixed occupancy in each configuration. Such an expansion (suppressing the spin functions for simplicity) takes the form:

$$
\begin{gathered}
\Psi(1, \ldots N)=\sum_{i, j} C_{i, j} \mid \varphi_{n_{1}}(1) \varphi_{n_{2}}(2) \ldots \varphi_{n_{N-2}}(N-2) \\
\quad \times \chi_{i}\left(r_{N-1}\right) Y_{l_{i} m_{i}}\left(\Omega_{N-1}\right) \chi_{j}\left(r_{N}\right) Y_{l_{j} m_{j}}\left(\Omega_{N}\right) \mid .
\end{gathered}
$$

The radial grid is partitioned into a number of finite elements, with DVR basis functions in each element. We use this underlying DVR basis to form a set of atomic orbitals, which are themselves constructed as linear combinations of DVR functions, but only using the M DVR functions that span the first few elements:

$$
\varphi_{\alpha}(r)=\sum_{j=1}^{M} U_{\alpha j} \chi_{j}(r) .
$$

Since the primitive DVR polynomial basis functions have compact support within the finite element boundaries, 
the entire basis of orbitals and DVR functions can be kept orthonormal. This hybrid approach, as we have shown, takes advantage of a key simplification in the calculation of two-electron integrals when using FEM-DVR basis functions, namely, that the radial portion of the electron-electron repulsion matrix elements are diagonal in the DVR index of each electron $[4,5]$.

When the core electrons are constrained to doubly occupy a set of orbitals, the effective two-electron Hamiltonian is particularly simple and takes the form:

$$
H=h(1)+h(2)+\frac{1}{r_{12}},
$$

where $1 / r_{12}$ is the Coulomb repulsion between the active electrons and the one-body operator $h$ is

$$
h=T-\frac{Z}{r}+\sum_{\mathrm{o}} 2 J_{\mathrm{o}}-K_{\mathrm{o}},
$$

where $T$ is the one-electron kinetic energy operator, $Z / r$ the nuclear attraction, the sum runs over all the doubly occupied orbitals and $2 J_{\mathrm{o}}$ and $K_{\mathrm{o}}$ are the direct and exchange components, respectively, of the core interaction with the active electrons. Eqs. (3)-(4) apply directly to the case of $\mathrm{Be}$, where the core consists of the doubly occupied $1 s$ orbital.

When a core orbital is to be only partially occupied in each configuration, a frozen core ansatz is still well defined by using Eq. (1) and giving that core orbital the same partial occupancy in each configuration, but it is then not possible to uniquely define an energyindependent effective two-electron Hamiltonian without further approximation [6]. This is the case for the 3electron lithium atom, when we constrain one of the core electrons to occupy a $1 s$ orbital, while allowing the other two electrons to be unconstrained. An effective 2-electron Hamiltonian for lithium is obtained by starting with a frozen core ansatz for the 3-electron wave function:

$$
\Psi(1,2,3)=\mathcal{A}\left[\Psi(12) \varphi_{1 s}(3)\right],
$$

where $\Psi(12)$ is presumed to be antisymmetric and $\mathcal{A}$ antisymmetrizes the coordinates of electron 3 with those of 1 and 2. If we ignore exchange scattering with the frozen core electron, i.e. assume $\mathcal{A}=1$, then we can substitute Eq. (5) into the Schrödinger equation and integrate out the coordinates of electron 3 to obtain an effective twoelectron Hamiltonian as in Eq.(3), where the one-body operators are now given as

$$
h(r)=T-\frac{3}{r}+J_{1 s}(r) .
$$

One could attempt to refine the ansatz by including a semi-empirical local exchange potential in Eq. (6) [7], but we have not done so here. We will refer to the combination of Eqs. (3) and (6) as an effective potential frozen-core approximation, to distinguish it from a frozen-core expansion with full exchange. The frozencore models, both with and without full exchange, have been applied to DPI from ground-state lithium [8, 9] as well as low-energy electron-impact ionization of helium [7]. The models were found to give results that were in good mutual accord and that agreed with available experimental data $[8,10]$.

Some care is needed when working with an unconstrained primitive DVR basis and an effective twoelectron Hamiltonian to surpress the appearance of unphysical (bosonic) states that result from over-population of core orbitals by more than two electrons. In our hybrid orbital-DVR approach, for symmetries in which there are core orbitals, the atomic orbitals are, by construction, orthogonal to the other primitive DVR functions used in the representation of the effective Hamiltonian, so the imposition of proper orthogonality constraints is straightforward. For the target atoms considered here, we can use the complete basis of primitive DVRs for all basis functions with $l \neq 0$, since there are no restrictions in those symmetries. For calculations on beryllium, we must exclude all two-electron configurations involving a $1 s$ orbital, since it is doubly occupied in the core, while for lithium, only one configuration, namely $1 s^{2}$ is excluded from the expansion.

The amplitude for double photoionization is constructed from a solution of the driven equation for the first-order wave function (scattered wave) that is obtained when the radiation field is treated as a perturbation:

$$
\left(E_{0}+\omega-H\right) \Psi_{s c}^{+}=(\boldsymbol{\epsilon} \cdot \boldsymbol{\mu}) \Psi_{0},
$$

where $H$ is the effective Hamiltonian as given above, $\omega$ is the photon frequency, $\epsilon$ is the photon polarization vector, $\boldsymbol{\mu}$ is the electronic dipole operator and $\Psi_{0}$ is the wave function describing the initial state of the atom with energy $E_{0}$. Equation (7) must be solved with outgoingwave boundary conditions. Those boundary are rigorously applied by transforming the radial coordinates of the electrons according to the exterior complex scaling (ECS) transformation, which scales those coordinates by a phase factor beyond some radius $R_{0}$, as discussed for example in [5]. The scattered wave $\Psi_{\mathrm{sc}}^{+}$is expanded in coupled spherical harmonics,

$$
\Psi_{\mathrm{sc}}^{+}=\sum_{l_{1} l_{2}} \frac{\psi_{l_{1}, l_{2}}\left(r_{1}, r_{2}\right)}{r_{1} r_{2}} \mathcal{Y}_{l_{1}, l_{2}}^{L M}\left(\hat{\mathbf{r}}_{1}, \hat{\mathbf{r}}_{2}\right),
$$

and then used to compute the amplitude $f\left(\mathbf{k}_{1}, \mathbf{k}_{2}\right)$ for double photoionization producing photolectrons with momenta $\mathbf{k}_{1}$ and $\mathbf{k}_{2}$.

The DPI amplitude can be expressed as a coherent sum of partial-wave amplitudes $[11,12]$ :

$$
\begin{aligned}
f\left(\mathbf{k}_{1}, \mathbf{k}_{2}\right)= & \sum_{l_{1}, l_{2}}\left(\frac{2}{\pi}\right) i^{-\left(l_{1}+l_{2}\right)} e^{i \eta_{l_{1}}\left(k_{1}\right)+i \eta_{l_{2}}\left(k_{2}\right)} \\
& \times \mathcal{F}_{l_{1}, l_{2}}\left(k_{1}, k_{2}\right) \mathcal{Y}_{l_{1}, l_{2}}^{L M}\left(\hat{\mathbf{k}}_{1}, \hat{\mathbf{k}}_{2}\right),
\end{aligned}
$$

where the radial amplitudes $\mathcal{F}_{l_{1}, l_{2}}\left(k_{1}, k_{2}\right)$, which are evaluated along a hypersphere $\rho_{0}$ in the $\left(r_{1}, r_{2}\right)$-plane just 
inside the ECS turning point $R_{0}$, are computed using a surface-integral formulation that amounts to the integration of the Wronskian between the scattered wave decomposition of the full solution $\psi_{l_{1}, l_{2}}\left(r_{1}, r_{2}\right)$ and two partial-wave testing functions $[5,11,12]$ :

$$
\begin{aligned}
& \mathcal{F}_{l_{1}, l_{2}}\left(k_{1}, k_{2}\right)= \\
& \frac{\rho_{0}}{2} \int_{0}^{\pi / 2}\left[\varphi_{l_{1}}^{k_{1}}\left(r_{1}\right) \varphi_{l_{2}}^{k_{2}}\left(r_{2}\right) \frac{\partial}{\partial \rho} \psi_{l_{1}, l_{2}}\left(r_{1}, r_{2}\right)\right. \\
& \left.-\psi_{l_{1}, l_{2}}\left(r_{1}, r_{2}\right) \frac{\partial}{\partial \rho} \varphi_{l_{1}}^{k_{1}}\left(r_{1}\right) \varphi_{l_{2}}^{k_{2}}\left(r_{2}\right)\right]\left.\right|_{\rho=\rho_{0}} d \alpha,
\end{aligned}
$$

where the integration is over the hyperspherical angle $\alpha \equiv \tan ^{-1}\left(r_{2} / r_{1}\right)$. The testing functions $\varphi_{l}^{k}$ themselves are partial-wave components of the continuum eigenfunctions of the one-body operator $h$ defined in Eq. (3). Their numerical construction is detailed in ref. [4].

The triply differential cross sections (TDCS) are computed from the amplitudes in Eq. 9 by

$$
\frac{d^{3} \sigma}{d E_{1} d \Omega_{1} d \Omega_{2}}=\frac{4 \pi^{2} \omega}{c} k_{1} k_{2}\left|f\left(\mathbf{k}_{1}, \mathbf{k}_{2}\right)\right|^{2}
$$

in the length gauge. Integration of the TDCS over the angles $\Omega_{1}$ and $\Omega_{2}$ of the electrons yields the singly differential cross section (SDCS) which is given simply by

$$
\frac{d \sigma}{d E_{1}}=\frac{4 \pi^{2} \omega}{c} k_{1} k_{2}\left(\frac{2}{\pi}\right)^{2} \sum_{l_{1} l_{2}}\left|\mathcal{F}_{l_{1}, l_{2}}\left(k_{1}, k_{2}\right)\right|^{2}
$$

With this definition of the SDCS, the total integrated cross section for double photoionization at photon energy $\omega$ with excess energy $E=\omega+E_{0}$ is

$$
\sigma=\int_{0}^{E} \frac{d \sigma}{d E_{1}} d E_{1}
$$

Since the SDCS is symmetric about $E / 2$, one can alternatively define the total cross section as

$$
\sigma=\int_{0}^{E / 2} \frac{d \widetilde{\sigma}}{d E_{1}} d E_{1}
$$

where the scaled SDCS is defined as

$$
\frac{d \widetilde{\sigma}}{d E_{1}}=2 \frac{d \sigma}{d E_{1}}
$$

so that the total DPI cross section is computed by integrating the scaled cross section over half the available energy range. This definition is commonly used in previous theoretical work, so we continue to use the latter definition of the SDCS here.

\section{COMPUTATIONS}

For the calculations on $\mathrm{Li}$ and Be, the driven equation for the scattered wave was solved with radial grids that

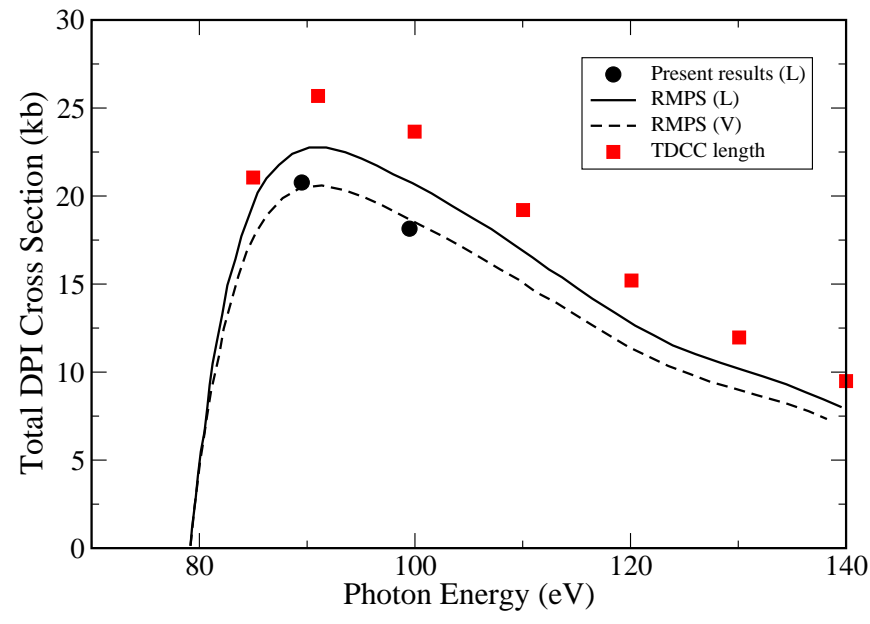

FIG. 1: (Color online) Total DPI cross section of lithium from the $1 s^{2} 2 p,{ }^{2} \mathrm{P}$ excited state. RMPS and TDCC results from ref. [8]. $1 \mathrm{~kb}=10^{-21} \mathrm{~cm}^{2}$.

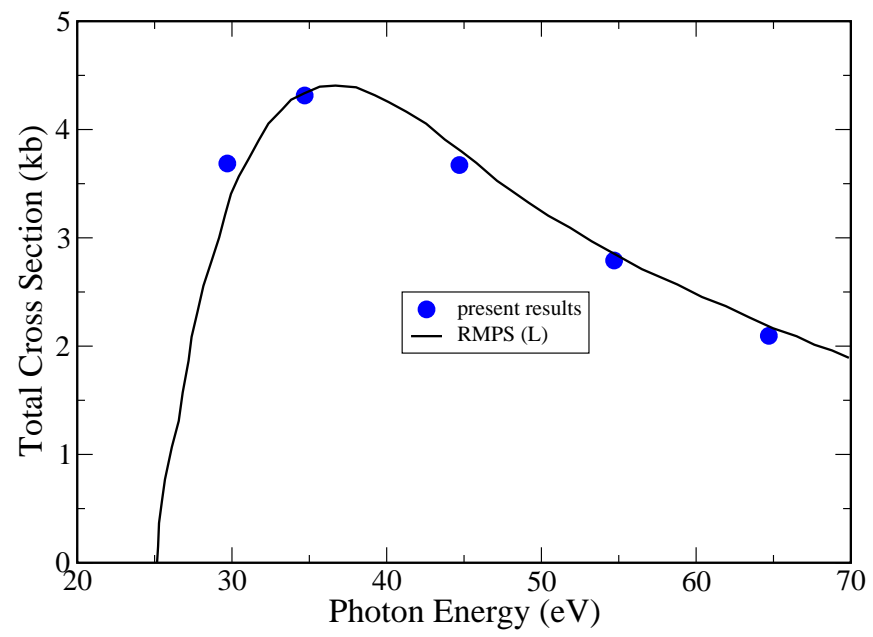

FIG. 2: (Color online) Total DPI cross section of beryllium from the $1 s^{2} 2 s 2 p,{ }^{3} \mathrm{P}$ excited state. RMPS results from ref. [13].

extended to 70.0 bohr and had finite-element boundaries at 2.0, 7.0,12.0, 20.0, 30.0, 40.0 and 50.0 bohr. We used 15th-order DVR in each element. The complex turning point $R_{0}$ was located at 40.0 bohr and the rotation angle was $30^{\circ}$. Partial waves up to $l_{\max }=9$ were included in the expansions. The $1 s$ orbital, along with a complementary set of atomic $s$-type atomic orbitals, was formed using DVR functions only from the first two elements. The initial-state $(1 s) 1 s 2 p,{ }^{2} \mathrm{P}$ and $\left(1 s^{2}\right) 2 s 2 p,{ }^{1,3} \mathrm{P}$ target states for $\mathrm{Li}$ and $\mathrm{Be}$, respectively, were obtained from full configuration-interaction calculations with the effective 2-electron Hamiltonian using functions defined on the real portion of the radial grid out to $20.0 \mathrm{bohr}$.

For purposes of comparison, we will also show results for $1 s 2 p,{ }^{1,3} \mathrm{P}$ helium. The bound states were described on 4 finite elements with boundaries at 5.0, 10.0, 18.0, and 

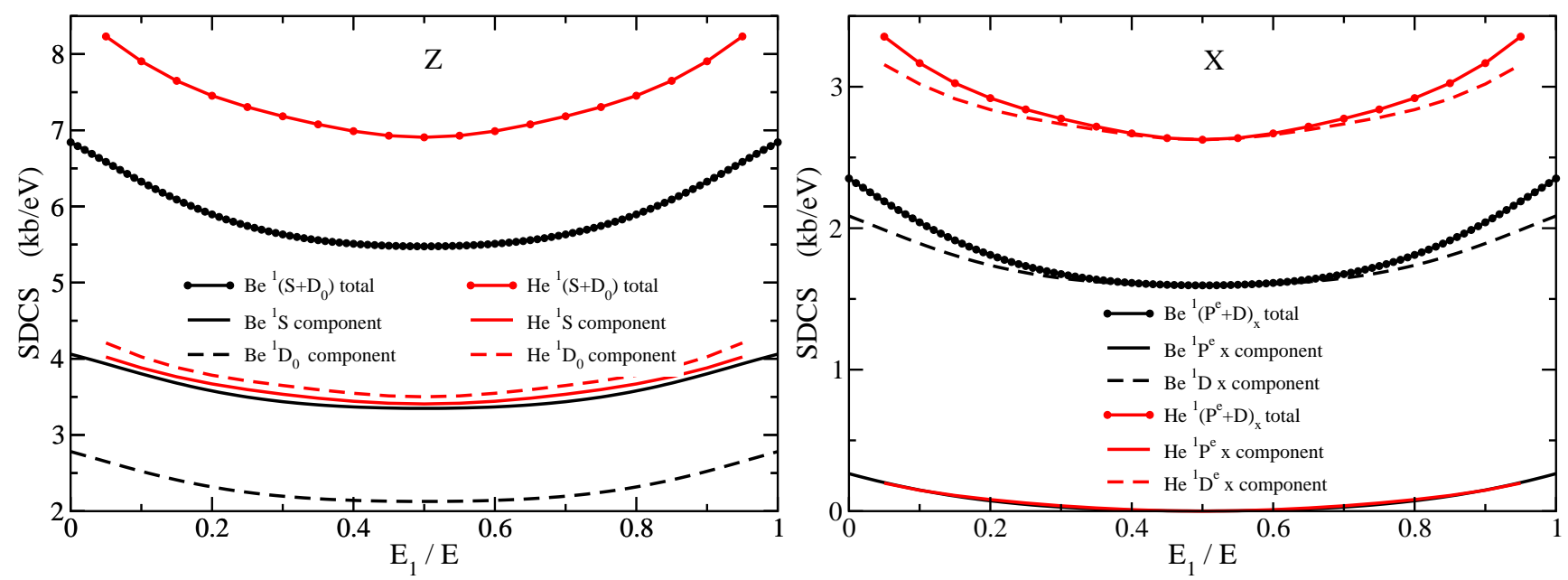

FIG. 3: (Color online) Singly differential cross sections for double ionization of ${ }^{1} \mathrm{P}_{0}$ beryllium (solid curves) and ${ }^{1} \mathrm{P}_{0}$ helium (dashed curves) at $10 \mathrm{eV}$ above threshold. Left panel: z-polarization; right panel: x-polarization.
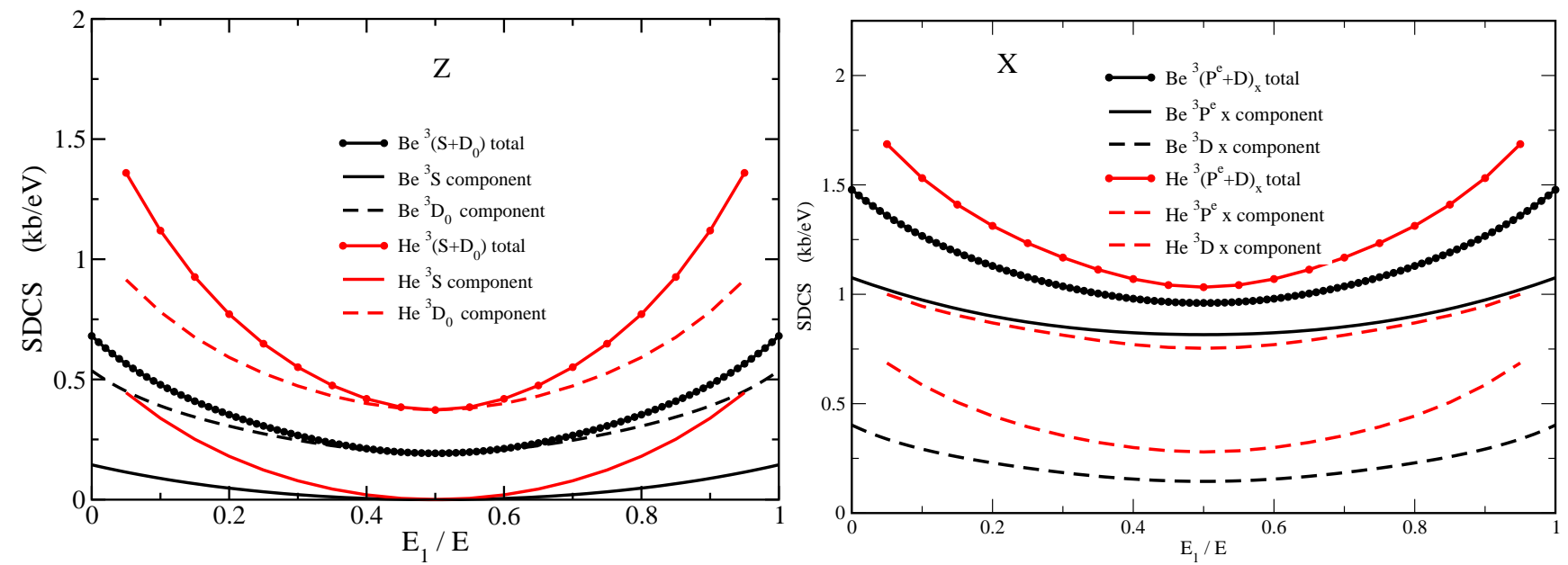

FIG. 4: (Color online) As in Fig. 3, for ${ }^{3} \mathrm{P}_{0}$ beryllium and ${ }^{3} \mathrm{P}_{0}$ helium.

26.0 bohr, while the continuum state calculations used 4 additional finite-element boundaries at 34.0, 42.0, 54.0 and 70.0 bohr with the ECS turning point at $R_{0}=42.0$ bohr. We again used $l_{\max =9}$ for these calculations.

We are confident that the results given below are converged with respect to grid size, DVR order, extraction radius and number of partial waves included. This was confirmed with calculations using fewer functions than those reported. We have also checked that calculations in the length and velocity gauges gave virtually identical results, so only length gauge results will be presented.

In all cases considered, we can perform separate calculations, in symmetry, using the 2-electron effective potential formulation with the two photoelectrons coupled to overall singlet or triplet states. In the Be and He cases, these double continua are reached from initial states of the same spin and opposite parity. In the Li case, the overall spin of the 3-electron system is a doublet, so one must combine the 2-electron singlet or triplet states with the remaining $1 s$ electron to make an overall doublet [7]. Since the spins of the photoejected electrons are generally not detected, we combine the two independent doublets using appropriate spin statistical weights to obtain the physical cross sections for $\mathrm{Li}[8]$ :

$$
\sigma_{\text {total }}=2\left(\frac{1}{4} \sigma_{S=0}+\frac{3}{4} \sigma_{S=1}\right)
$$

\section{RESULTS}

Total double ionization cross sections for the excited $1 s^{2} 2 p,{ }^{2} \mathrm{P}$ state of Li have been previously calculated using the R-matrix plus pseudostates (RMPS) and timedependent close-coupling (TDCC) methods [8] and are compared with the present results in Fig. 1. The present results agree rather well with the RMPS velocity gauge 

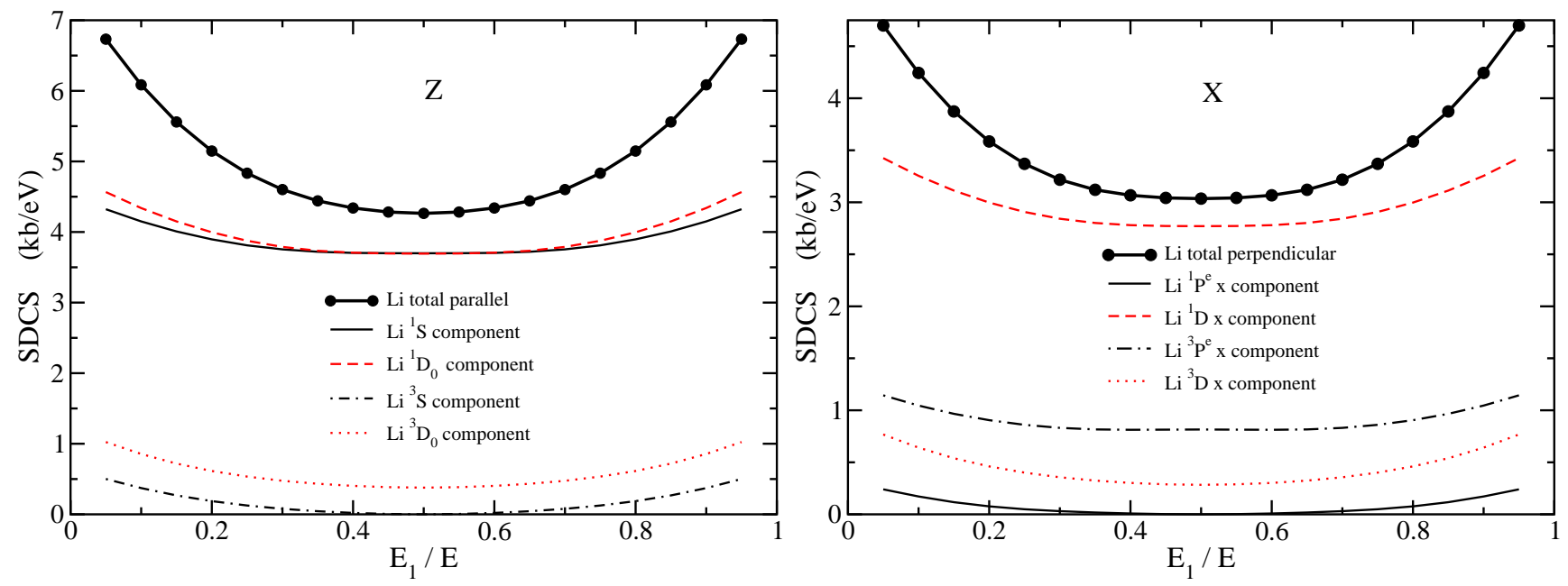

FIG. 5: (Color online) Singly differential cross sections for double ionization of ${ }^{2} \mathrm{P}_{0}$ lithium at 10 eV above threshold. Singlet (solid curves) and triplet (dashed curves) contributions are individually labelled. Left panel: z-polarization; right panel: $\mathrm{x}$-polarization.
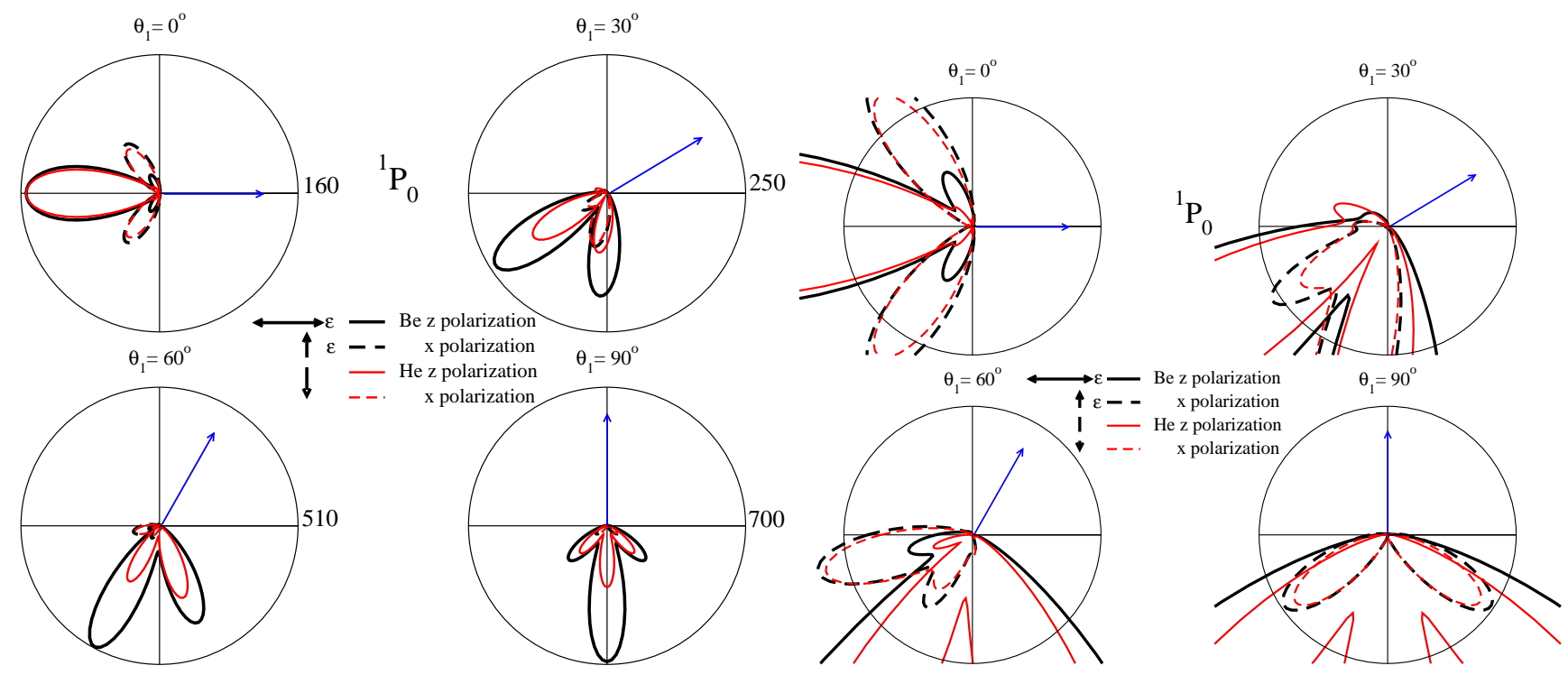

FIG. 6: (Color online) Triply differential cross sections for double ionization of ${ }^{1} \mathrm{P}_{0}$ beryllium (solid lines) and ${ }^{1} \mathrm{P}_{0}$ helium $($ dashed lines) with z- and x-polarized light at $10 \mathrm{eV}$ above threshold and equal energy sharing. The arrow indicates the direction of the fixed electron. Radii of circles, marked to the right, give magnitude of cross sections in units of b/eV/ $\mathrm{sr}^{2} \cdot 1 \mathrm{~b}=10^{-24} \mathrm{~cm}^{2}$. The right panels show a blowup of the same TDCS in more detail.

results, but are smaller than the RMPS length and TDCC results by approximately $10 \%$ and $20 \%$, respectively. RMPS total DPI cross sections are also available [13] for the $1 s^{2} 2 s 2 p,{ }^{3} \mathrm{P}$ state of Be and are compared with the present results in Fig. 2. In this case, the (polynomial fitted) RMPS length and velocity gauge results are almost identical and agree well with the present results.

Differential DPI cross sections for excited-state Li and Be have neither been measured nor previously calculated. For ground-state Li, TDCS results from TDCC and convergent close-coupling (CCC) calculations have recently been published [9]. We have compared those results with values obtained using the current methodology. We find excellent agreement with the TDCC results, and somewhat poorer agreement with CCC, to the extent that the latter two show small differences.

Before proceeding to the differential cross sections, it is useful to review the dipole selection rules when the initial state of the target atom has $\mathrm{P}^{o}$ symmetry. Without loss of generality, we can choose the z-direction as the axis of quantization and assume the initial state is aligned along that direction, i.e, it has quantum numbers $\mathrm{L}=1$ and $\mathrm{M}=0$. We then consider ionization by photons lin- 


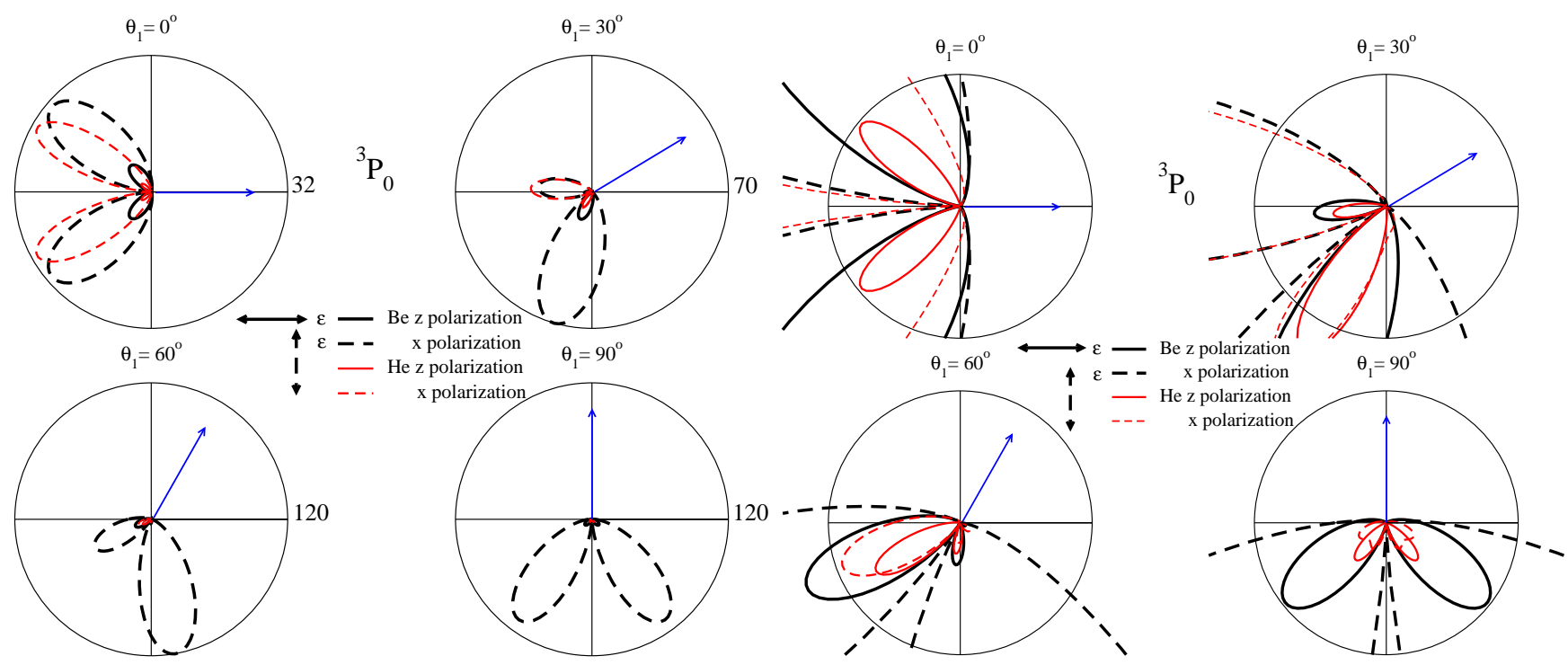

FIG. 7: (Color online) As in Fig. 6, for ${ }^{3} \mathrm{P}_{0}$ beryllium and ${ }^{3} \mathrm{P}_{0}$ helium.
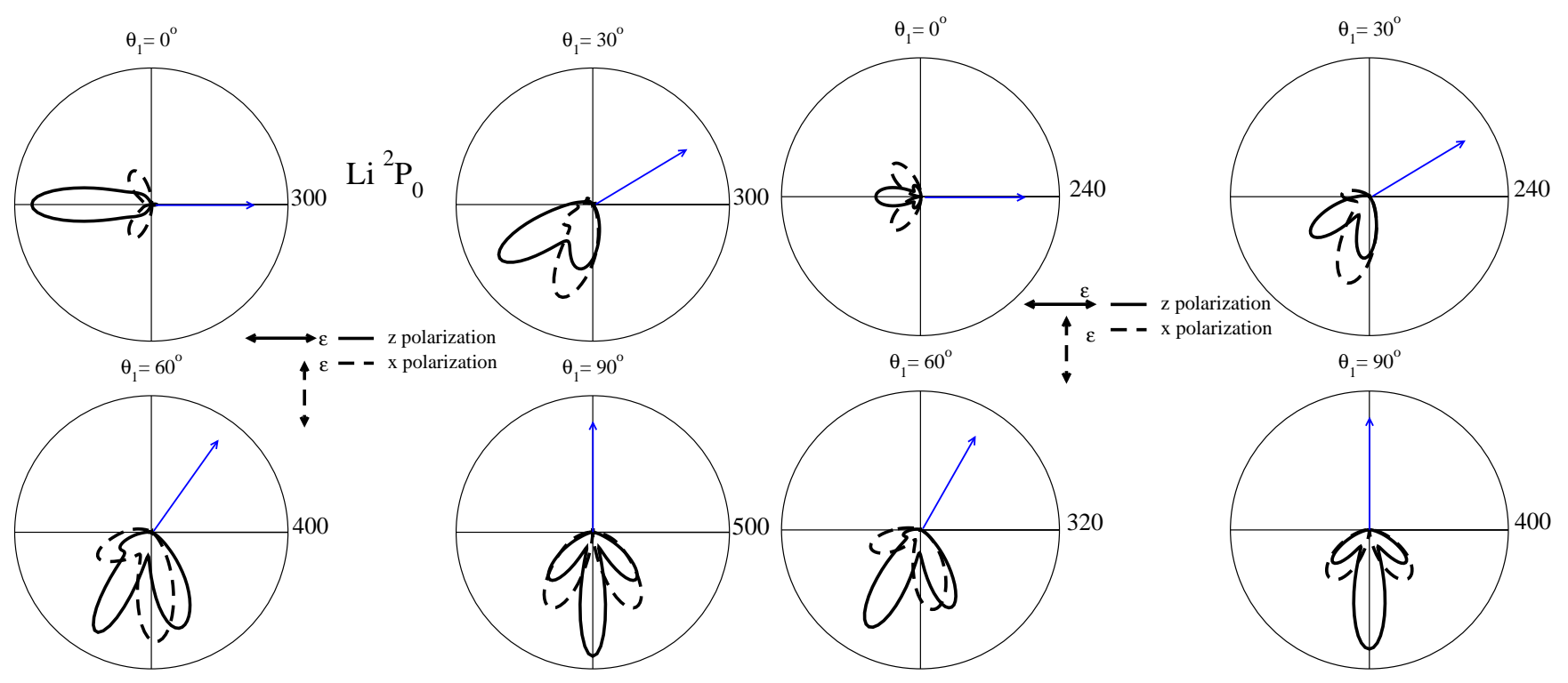

FIG. 8: (Color online) Four left panels: triply differential cross sections for double ionization of ${ }^{2} \mathrm{P}_{0}$ lithium with $\mathrm{z}-$ and $\mathrm{x}-$ polarized light at $10 \mathrm{eV}$ above threshold and equal energy sharing. The four right panels show TDCS using beryllium data, but combined as in they would be for lithium (see text). Radii of circles, marked to the right, give magnitude of cross sections in units of $\mathrm{b} / \mathrm{eV} / \mathrm{sr}^{2}$.

early polarized along the $\mathrm{x}$ or $\mathrm{z}$ directions. For parallel (z) polarization, the final continuum states are connected to the initial state by the z-component of the dipole operator and can have $\mathrm{S}$ or $\mathrm{D}_{0}^{e}$ symmetry. For perpendicular (x) polarization, the final continuum states are connected to the initial state by the $\mathrm{x}$-component of the dipole operator and can have $\mathrm{D}_{ \pm 1}^{e}$ or $\mathrm{P}_{ \pm 1}^{e}$ symmetry. $\mathrm{P}^{o} \rightarrow \mathrm{P}^{e}$ transitions are dipole-forbidden with one-electron targets, but are fully allowed in many-electron atoms. Transitions to the $\mathrm{P}^{e}$ continua are not mentioned in the two recent studies of DPI from ${ }^{2} \mathrm{P}$ excited $\mathrm{Li}[2,8]$. We note that while there is no selection rule that can be used to predict the ratio of integral cross sections (either total or SDCS) for parallel to perpendicular polarization, since more than one total symmetry component is involved, a straightforward application of the Wigner-Eckart theorem shows that the ratio of $\mathrm{D}_{0}$ to $\mathrm{D}_{x}$, for both total cross sections and SDCS at all energy sharings, must be $4 / 3$.

The SDCS for $\mathrm{Be}^{1} \mathrm{P}_{0}$ and ${ }^{3} \mathrm{P}_{0}$ at $10 \mathrm{eV}$ excess energy above threshold are shown in Figs. 3 and 4, respectively. For comparison, we also plot results for the same excited He states at the same excess energy. The He cross sections are generally larger than the corresponding Be cross sections, but the relative contributions of the vari- 
ous symmetry components to the total are similar. Several selection rules are evident in the results, namely, that the ${ }^{3} \mathrm{~S}$ and ${ }^{1} \mathrm{P}^{e}$ contributions vanish at equal energy sharing [14]. Curiously, the $\mathrm{P}^{e}$ component, which only contributes to DPI with perpendicular polarization, is vanishingly small at all energy sharings in the case of the ${ }^{1} \mathrm{P}_{0}$ targets, but is the dominant component in the case of the ${ }^{1} \mathrm{P}_{0}$ targets. We note the ratio of $\mathrm{D}_{0}$ to $\mathrm{D}_{x}$ is always $4 / 3$.

Figure 5 shows SDCS for $\mathrm{Li}^{2} \mathrm{P}_{0}$ at $10 \mathrm{eV}$ excess energy. For each allowed spatial symmetry, we show the individual spin contributions, as well s the totals, the latter computed using Eq. (16). The relative magnitudes of the various components is somewhat different for $\mathrm{Li}$ than it is for Be. With parallel polarization, ${ }^{1} \mathrm{~S}$ and ${ }^{1} \mathrm{D}$ components are almost the same, where as in Be, ${ }^{1} \mathrm{~S}$ is the dominant. The triplet components are generally smaller than the singlet contributions, a fact also noted by Colgan et al. [8].

Figures 6 and 7 show TDCS for the ${ }^{1} \mathrm{P}_{0}$ and ${ }^{3} \mathrm{P}_{0}$ states of beryllium, respectively. We have again plotted the TDCS for the analogous states of He for comparison. The He singlet and triplet results were results scaled, separately in both cases, so that the major lobes at $\Theta_{1}=0^{\circ}$ had the same peak values as in Be. Even with this internormalization, the He and Be TDCS are found to be rather different, apart from the general pattern of lobes and zeros which are largely dictated by symmetry. It is evident that the angular distributions are very sensitive to the orientation of the polarization. In all cases, we observe that the "B1" selectrion rule [14] is satisfied, which states that both electrons can't go out perpendicular to the polarization direction for final state $M$ not equal to zero. This is evident for the $\Theta_{1}=0^{\circ}$ panels in Figs. 6 and 7 for x-polarization. We can see that no such selection rule applies when the final state has $\mathrm{M}=0$, by examining the z-polarization results for $\Theta_{1}=90^{\circ}$ in both Figs. 6 and 7. For the triplet cases shown in Fig. 7, we see another selection rule at play which prevents backto-back ejection, independent of the orientation of the polarization. Note that for these equal energy sharing results, the two photoelectrons, due to Coulomb repulsion, are never ejected in the same direction.

Finally, we show TDCS results for ${ }^{2} \mathrm{P}_{0}$ lithium in Fig. 8. Since these are obtained from an incoherent com- bination of singlet and triplet contributions, the only selection rules that survive are those preventing back to back ejection for x-polarization at $\Theta_{1}=0^{\circ}$ and $90^{\circ}$. To highlight underlying similarities between the TDCS for Be and Li, we have taken the TDCS for singlet and triplet Be and combined them as we would for Li, using Eq. 16. The TDCS for this "synthetic" Li atom, constructed from Be results, are shown in the right-hand panels of Fig. 8. They bear a marked similarity to the true Li results, particularly at $\Theta_{1}=60^{\circ}$ and $90^{\circ}$.

\section{CONCLUSIONS}

The hybrid orbital/DVR method offers an efficient and viable way of extending grid-based studies of DPI to many-electron atoms with two active electrons. We have applied this technique to obtain fully differential cross sections from aligned, excited P-states of lithium and beryllium and have shown that the TDCS are very sensitive to the orientation dependence of the ionizing radiation with respect to the axis of quantization. Comparisons between Be and analogous helium states shows that the differences are much larger when comparing excitedstate cross sections than ground-state cross sections. Finally we have been able to show that similarities between Be and Li can be revealed when the TDCS for singlet and triplet excited $\mathrm{Be} \mathrm{P}$-states are incoherently combined to produce cross sections for a "synthetic" Li atom. It is our hope that these results will prompt future experiments aimed at kinematically complete measurements of DPI with aligned atomic targets, thereby extending the body of such information that currently includes only ground-state targets.

\section{Acknowledgments}

This work was performed under the auspices of the US Department of Energy by the Los Alamos National Laboratory and the University of California Lawrence Berkeley National Laboratory under Contract DE-AC0205CH11231 and was supported by the U.S. DOE Office of Basic Energy Sciences, Division of Chemical Sciences. CWM acknowledges support from the National Science Foundation (Grant No. PHY-0604628).
[1] J. Colgan and M. S. Pindzola, Phys. Rev. A 67, 012711 (2003).

[2] G. Zhu, M. Schuricke, J. Steinmann, J. Albrecht, J. Ullrich, I. Ben-Itzhak, T. J. M. Zouros, J. Colgan, M. S. Pindzola, and A. Dorn, Phys. Rev. Lett. 103, 103008 (2009).

[3] T. N. Rescigno and C. W. McCurdy, Phys. Rev. A 62, 032706 (2000).

[4] F. L. Yip, C. W. McCurdy, and T. N. Rescigno, Phys. Rev. A 81, $\operatorname{xxxx}(2010)$.
[5] C. W. McCurdy, M. Baertschy, and T. N. Rescigno, J. Phys. B 37, R137 (2004).

[6] M. E. Riley and D. G. Truhlar, J. Chem. Phys. 65, 792 (1976).

[7] M. S. Pindzola and F. J. Robicheaux, Phys. Rev. A 61, 052707 (2000).

[8] J. Colgan, D. C. Griffin, C. P. Ballance, and M. S. Pindzola, Phys. Rev. A 80, 063414 (2009).

[9] A. S. Kheifets, D. V. Fursa, C. W. Hines, I. Bray, J. Colgan, and M. S. Pindzola, Phys. Rev. A 81, 023418 (2010). 
[10] J. Colgan, M. S. Pindzola, G. Childers, and M. A. Khakoo, Phys. Rev. A 73, 042710 (2006).

[11] D. A. Horner, J. Colgan, F. Martín, C. W. McCurdy, M. S. Pindzola, and T. N. Rescigno, Phys. Rev. A 70, 064701 (2004).

[12] F. L. Yip, D. A. Horner, C. W. McCurdy, and T. N.
Rescigno, Phys. Rev. A 75, 042715 (2007).

[13] D. C. Griffin, M. S. Pindzola, C. P. Ballance, and J. Colgan, Phys. Rev. A 79, 023413 (2009).

[14] F. Maulbetsch and J. S. Briggs, J. Phys. B 28, 551 (1995). 